\title{
LES MOOKS \\ OU LE NOUVEAU RETOUR DU RÉCIT EN JOURNALISME
}

\author{
Marie Vanoost ${ }^{1}$
}

\begin{abstract}
Les mooks se présentent comme des objets médiatiques novateurs, tant dans leur format et leur identité graphique que dans leur modèle économique, tout en invoquant comme références les longues traditions du grand reportage à la française et du narrative journalism à l'américaine. Cet article se propose de revenir sur quelques grands traits de ces deux traditions narratives, afin de mieux comprendre l'héritage que revendiquent les mooks. Il fait ainsi apparaître à quel point ces nouveaux objets s'inscrivent, par le recours au récit, dans une longue et complexe histoire de résistance aux pratiques journalistiques dominantes.
\end{abstract}

Les mooks, revues à mi-chemin entre le magazine et le livre qui se sont récemment multipliées dans le paysage médiatique d'Europe francophone, se présentent comme des objets médiatiques volontairement novateurs, tout en développant un fort discours de différenciation par rapport au reste de la presse actuelle. Alors que l'information se fait de plus en plus courte et rapide, la plupart des mooks veulent revenir à l'épaisseur d'un récit long, qui prend son temps, dans la lignée à la fois du grand reportage à la française et du narrative journalism à l'américaine.

Sil'on a pu dire du premier qu'il mise " sur toutes les ressources qui sont celles du roman » (Martin, 2005, p. 176) et du second

1 Marie Vanoost est chargée de recherches du F.R.S.-FNRS à l'Université catholique de Louvain au sein de l'Observatoire de Recherche sur les Médias et le journalisme (ORM).

Recherches en communication, n46 - Article publié le 24/04/2018 
qu'il se lit « comme un roman » (Wolfe, 1975, p. 21), il s'agit de deux traditions journalistiques distinctes qui ont été étudiées dans des perspectives différentes. Cependant, puisqu'elles sont toutes deux mises en avant comme modèles par les mooks, cet article se propose de revenir sur quelques grands traits de ces deux traditions, afin de les mettre en perspective et en dialogue, faisant ainsi apparaître comment, au-delà la nouveauté affichée dans leur matérialité, les mooks s'inscrivent dans une longue tradition de résistance aux modèles journalistiques dominants.

\section{Les mooks, entre nouveauté et héritage}

Les mooks - contraction des mots anglais magazine et bookse sont développés en Europe francophone dans le sillage de la revue $X X I$, lancée en 2008 . On a ainsi vu naître - et parfois déjà mourir - 6 Mois, Feuilleton, Desports, Long Cours, La Revue dessinée, 24h01, etc. Ces revues se présentent comme des objets médiatiques originaux : un rythme de parution tri- ou semestriel, plusieurs centaines de pages pour une quinzaine d'euros, pas de publicité, une identité graphique forte et audacieuse.

Ces nouveaux supports se distinguent également par la revendication d'un modèle journalistique radicalement différent de celui qui domine actuellement la sphère journalistique. S'opposant à l'accélération et à l'amplification que connaît l'information - principalement sous la pression d'Internet -, la plupart des mooks revendiquent un retour au récit, au format long et au temps lent. Leurs discours apparaissent teintés d'une certaine nostalgie et tournés au moins autant vers le passé que vers le présent et l'avenir, qu'ils espèrent meilleur, du journalisme. Le premier éditorial de $X X I$ s'ouvre d'ailleurs sur une citation du célèbre grand reporter Albert Londres :

«Messieurs, vous apprendrez qu'un reporter ne connaît qu'une ligne, celle du chemin de fer ", répliqua Albert Londres en claquant la porte du Quotidien, le journal qui l'employait et dont les dirigeants lui reprochaient de ne pas être «dans la ligne ». 
Ces mots nous les faisons nôtres au moment de lancer le premier numéro de $X X I$, dont le titre $-X X I$ comme $\mathrm{XXI}^{\mathrm{e}}$ siècle - montre notre volonté de prendre l'époque à bras-le-corps. [...]

Ce journalisme est éternel, seules ses formes changent. Il est toujours aussi nécessaire. L'information s'est multipliée, et notre regard s'est rétréci. Prendre le temps, se décaler, redonner des couleurs au monde, de l'épaisseur aux choses, de la présence aux gens, aller voir, rendre compte : telle est la volonté de XXI. (XXI, $\mathrm{n}^{\circ} 1,2007$, p. 1)

On retrouve cette dialectique entre présent et passé dans de nombreux mooks. S'inspirant du New Yorker (fondé en 1925) et de Granta (dont la formule actuelle remonte à la fin des années 1970), la revue Feuilleton propose principalement des traductions de grands journalistes narratifs américains, contemporains ou plus anciens, remontant même jusqu'à la fin du $19^{\mathrm{e}}$ siècle et une des figures pionnières de cette tradition, Nellie Bly. L'éphémère Ithaque, qui se voulait « un journal papier 2.0 », selon le titre de son premier éditorial, identifiait certains de ses articles comme des « reportages gonzo », en référence au mouvement américain des années 1960-70. Durant sa courte existence, Ithaque a également adapté en bande dessinée le grand reportage "Chez les fous » d'Albert Londres, paru en 1925. Sur son site web, la revue belge $24 h 01$ dénonce le contexte actuel d' « infobésité » et invoque trois références : Albert Londres, le New Yorker et la revue XXI.

$\mathrm{Si}$, comme l'écrit $X X I$, le journalisme que remettent en avant les mooks est « éternel », les fondateurs des différentes revues en dessinent néanmoins une histoire aux contours très différents. Patrick de Saint-Exupéry, rédacteur en chef de $X X I$ et fondateur de 6 Mois, déclare (lors d'un entretien réalisé le 28 janvier 2013) :

Je dis « journalisme narratif » parce que c'est pratique, mais sur le fond, narrative writing ou narrative journalism, c'est un concept que les Américains ont repris de la vieille Europe. [...] la base du journalisme narratif, c'est Albert Londres, c'est Kessel. Enfin voilà, ça vient d'Europe, ensuite c'est reparti aux États-Unis où là ça $\mathrm{a}$ été transformé en narrative writing et, comme ça vient des 
États-Unis, ça revient en Europe, mais à l'origine, c'est toujours venu d'ici.

Par contre, pour Adrien Bosc (dans un entretien réalisé le 6 juin 2013), directeur de publication de Feuilleton et Desports, les racines sont clairement américaines :

Pour qu'il y ait modèle, pour qu'il y ait tradition, il faut qu'il y ait des formats, dans un pays, assez forts pour qu'il y ait une émulation collective. Il se trouve qu'aux ÉtatsUnis il existe le New Yorker, il existe le journal Harper's, Esquire et je pourrais continuer. [...] Et ces quatre, cinq, six journaux œuvrent depuis soixante ans à élever le niveau du reportage. [...] Tout ça a créé sur des décennies une tradition qui n'existe pas ailleurs, ou très peu.

Les traditions journalistiques évoquées par les mooks, toutes deux fondamentalement narratives et interrogeant les frontières entre journalisme et littérature, ont été - et sont encore - étudiées dans des univers et des perspectives académiques différents ${ }^{2}$. Aux États-Unis, le journalisme narratif est considéré comme une forme ou un genre mettant en œuvre les techniques d'écriture de la fiction pour raconter des histoires réelles. Il fait l'objet d'un vaste travail d'historicisation et de canonisation (notamment Connery, 1992 ; Hartsock, 2000 ; Sims, 2007, 2008), de la part de spécialistes tant de la littérature que du journalisme ${ }^{3}$.

En France, plusieurs historiens et littéraires se sont récemment penchés sur l'histoire du journalisme pour cerner aussi

2 Il existe quelques ouvrages (Bak \& Reynolds, 2011 ; Keeble \& Tulloch, 2012, 2014) qui se réclament d'une perspective internationale sur le journalisme littéraire (sur la notion de journalisme littéraire : voir note suivante). Cependant, il s'agit principalement dans ces recueils d'articles d'appliquer les définitions existantes très largement américaines - à des productions journalistiques de différents pays, plutôt que de tenter d'établir des critères, définitions et théories plus globaux sur ce que représente le journalisme littéraire à une échelle internationale.

3 La plupart des travaux dans cette veine utilisent l'appellation journalisme littéraire, quelque peu plus restrictive que celle de journalisme narratif, mais qui en reste très proche, puisque la littérature est envisagée par ces auteurs uniquement sous l'angle de la fiction narrative (pour une discussion détaillée des différentes appellations : Vanoost, 2013). 
bien les façons dont la littérature imprègne le journal que celles dont, en retour, le journal imprègne la littérature (notamment Boucharenc \& Deluche, 2001 ; Kalifa et al., 2012 ; Thérenty, 2007 ; Thérenty \& Vaillant, 2004). Ces travaux s'intéressent au texte médiatique, incluant notamment les annonces ou le romanfeuilleton, là où les auteurs américains se focalisent sur le texte journalistique. Ils envisagent la littérature dans une optique large, comprenant les œuvres de fiction, mais aussi l'éloquence et l'art épistolaire, alors que les spécialistes américains tiennent globalement littérature et fiction narrative pour synonymes. Le grand reportage, en tant que récit - plus ou moins - littéraire de " choses vues », est l'un des genres étudiés dans cette veine de travaux ${ }^{4}$.

Toutes ces différences rendent délicate une comparaison de la tradition journalistique narrative américaine et de l'histoire $\mathrm{du}$ grand reportage français - d'autant plus que les sphères journalistiques française et américaine présentent chacune leurs spécificités et une évolution propre. En gardant à l'esprit ces limites et difficultés, on peut cependant tenter d'esquisser quelques liens possibles entre les deux traditions journalistiques invoquées par les mooks à titre de modèles, afin de mieux comprendre l'héritage que ces nouveaux objets médiatiques revendiquent.

\section{Grand reportage vs. journalisme narratif ?}

\subsection{Naissance}

Aux États-Unis, la naissance du journalisme narratif est généralement fixée à la fin du $19^{\mathrm{e}}$ siècle. Selon Robert Boynton, il s'agit d'un genre spécifiquement américain, né de la situation exceptionnelle des États-Unis à cette époque (Boynton, 2005, p. xxi). Pour John Hartsock, le basculement vers un journalisme factuel - qui n'est autre que le journalisme américain moderne (Schudson, 1978) -, à une époque de grands changements

4 On peut noter ici que le reportage, de manière plus large, a également fait l'objet d'un travail d'historisation dans une approche plus sociologique (voir notamment Ruellan, 2007). 
sociaux, aurait provoqué une crise épistémologique fondamentale. Certains journalistes auraient alors pris conscience, d'une part, de l'impossibilité devant laquelle ils se trouvaient de rendre compte de ce que Hartsock nomme, à la suite de Mikhaïl Bakhtine, " l'infinitude du présent » et, d'autre part, de la façon dont le journalisme moderne tend à aliéner toute subjectivité. C'est pourquoi Hartsock voit dans le journalisme narratif américain naissant une tentative " to narrow the distance between the alienated subjectivity and the indeterminate object in a narrative strategy opposite that of objectified versions of journalism » (2000, p. 42).

À la même époque, la presse française connaît un tournant similaire vers un journalisme plus factuel (Ferenczi, 1996), ce qui se traduit notamment par le développement de deux genres nouveaux : l'interview et le reportage. Si certains historiens de la presse française y voient l'influence des transformations qui ont lieu dans les médias américains, Pascal Durand soutient que « [s]i débattue à la fin du siècle, l'américanisation de la presse française tient largement du cliché et de la légende : le modèle du reportage ne vient pas tant d'ailleurs que d'en bas, c'est-àdire des bas-fonds du journalisme populaire » (2012, p. 1020). Selon Marie-Eve Thérenty, le reportage est même le genre où se développe un nouveau paradigme, à la fois journalistique et littéraire, spécifiquement français : la « chose vue ».

La chose vue est le compromis trouvé par le journal français pour obéir aux contraintes du nouveau régime d'information fondé sur le primat du fait, tout en respectant la tradition d'un journalisme littéraire. Le fait se retrouve bien au centre du journal, mais il n'est pas pour autant le signe d'une objectivité sans défaut, car il est pris en charge par une subjectivité omniprésente qui restaure le lien entre fait, sensation et écriture. Le journalisme français est un journalisme de subjectivité, où le journaliste, loin de s'effacer devant l'événement, constitue au contraire le prisme par lequel il est rendu. (Thérenty, 2007, p. 204) 
Ce paradigme peut être illustré, par exemple, par Jules Huret décrivant «L'incendie du Bazar de la Charité » à Paris en 1897 :

J'entre à mon tour.

L'odeur, l'odeur terrible et écœurante de chair brûlée persiste. L'effroyable vision de cauchemar ! Ces genoux aux os rompus, ouverts comme par des coups de serpe, ces bras arrondis autour de la tete dans des positions de désespoir surhumain [...]. Je m'attarde. Je repasse dix fois devant ces rangées de lamentables restes. Je voudrais m'en aller, et pourtant je demeure. (Huret, 1901, pp. 266-267)

Ce passage ne semble cependant pas relever d'un paradigme fondamentalement différent de celui qu'adopte l'écrivainjournaliste américain Stephen Crane, considéré outre-Atlantique comme l'un des premiers journalistes de la veine narrative, lorsqu'il décrit le conflit entre la Grèce et la Turquie, en 1897 également :

I had seen skirmishes and small fights, but this was my first big battle. The roll of musketry was tremendous. From a distance it was like tearing a cloth; nearer, it sounded like rain on a tin roof and close up it was just a long crash after crash. It was beautiful sound - beautiful as I had never dreamed. (Crane, 1984, p. 935)

Comme le décrit bien Karen Roggenkamp (2005), un certain nombre de journalistes narratifs de la première génération sont devenus célèbres non seulement en tant qu'auteurs, mais aussi en tant que personnages dans leurs propres récits.

D'autres journalistes narratifs américains de la même époque ont plutôt eu tendance à cacher leur subjectivité derrière le $i l$ d'un témoin anonyme - comme Abraham Cahan, décrivant la vie au Barge Office, où étaient rassemblés tous les immigrés arrivant à New York :

"We don't starve!" the eldest girl protested. "How can you live without a drop of soup? No wonder we look like 
corpses. Look how thin we are! There is not a drop of blood left in us, is there?"

As the visitor surveyed her plump, florid cheeks he felt like saying that there was, but he did not. (Cahan, 1998, p. 79)

On peut néanmoins trouver le même type de stratégie d'énonciation dans les premiers grands reportages français. Ainsi, Gaston Leroux a pu écrire, dans ses articles sur la révolution russe : «Ce qui frappe 1'observateur, c'est la passion de l'autoorganisation qui anime des collectivités multiples [...]»(dans Filteau, 2001, pp. 312-313).

Le grand reportage à la française et le journalisme narratif américain semblent dès lors tous deux naître comme une forme de résistance au journalisme factuel qui se développe à la fin du $19^{\mathrm{e}}$ siècle, cette résistance passant par une remise en avant - sous des formes et à des degrés divers - du caractère fondamentalement humain et subjectif du journalisme. Il s'agit d'une hypothèse qui nous semble stimulante à approfondir, dans une optique de comparaison entre les États-Unis et la France, mais aussi avec d'autres sphères journalistiques qui pourraient avoir connu un développement similaire.

\section{2. Évolution}

Après sa naissance à la fin du $19^{\mathrm{e}}$ siècle, le journalisme narratif a continué à se développer comme modèle alternatif aux pratiques dominantes tout au long de l'histoire du journalisme américain. Les experts identifient trois périodes particulièrement importantes dans ce développement (Connery, 1992 ; Sims, 2007) : les années 1930-1940, durant lesquelles on le désigne parfois par le terme de reportage (marquant une certaine différence avec le terme usuel anglais report) ; les années 1960-1970, sous l'étiquette du New Journalism; et la fin du $20^{\mathrm{e}}$ siècle où se popularise l'expression narrative journalism.

En France, après sa naissance à la fin du $19^{\mathrm{e}}$ siècle, le grand reportage connaît son apogée dans les années 1920-1930, avant de 
lentement décliner (Boucharenc, 2004 ; Martin, 2005). Les années 1970-1980 sont marquées par de nouvelles expérimentations narratives en journalisme, notamment au sein du premier Libération (Lits, 2008 ; Muhlmann, 2004) et du magazine Actuel, qui se réclame à la fois des New Journalists américains, d'Albert Londres et de Joseph Kessel (Joignot, 2008 ; Kervran \& Kien, 2010). Ces initiatives restent néanmoins fort ponctuelles, jusqu'à l'apparition des mooks à la fin des années 2000 .

Les périodes de développement des deux traditions journalistiques narratives, de part et d'autre de l'Atlantique, apparaissent donc largement similaires - avec parfois une décennie d'avance, parfois une décennie de retard d'une tradition sur l'autre. Si Hartsock (2000) souligne que ces différentes périodes sont des moments de crises pour la société américaine, il paraît essentiel de souligner que ce sont aussi de moments de bouleversements profonds non seulement pour la sphère journalistique américaine, mais aussi pour la sphère journalistique francophone.

Les années 1930 correspondent à une amplification $\mathrm{du}$ mouvement de professionnalisation du journalisme qui s'accompagne d'une cristallisation de l'idéal d'objectivité, mais aussi des premières remises en cause fortes de cet idéal (Delporte, 1995 ; Schudson, 1978). Les années 1960-1970 voient arriver de nouveaux acteurs médiatiques - comme Libération ou New Yorkqui, à leur tour, remettent en cause l'objectivité journalistique (Muhlmann, 2004 ; Pauly, 2014). La fin du $20^{\mathrm{e}}$ siècle et le début du $21^{\mathrm{e}}$, enfin, sont marqués par l'incroyable révolution que représente Internet pour la production et la diffusion de l'information - et en réaction à laquelle sont apparus non seulement les mooks, mais des plateformes comme longform.org et longreads.com.

Il me semble que l'on pourrait dès lors avancer l'hypothèse que le recours à une forme et des techniques d'écriture narratives serait, de manière plus large, une forme de résistance par rapport à une codification croissante et à une pratique de plus en plus normée du journalisme - il s'agirait en quelque sorte de ré-explorer les frontières avec la littérature aux moments où le journalisme dominant vise à s'en distancier. Cette hypothèse, qui prolonge la première hypothèse formulée quant à la naissance des deux 
traditions et qui appelle, elle aussi, à être vérifiée et approfondie, paraît tout aussi porteuse dans une perspective internationale plus large.

\subsection{Nature et frontières}

Dans ce qui constitue un des rares efforts à ce jour pour développer une perspective plus globale sur les différents types de rencontres entre récit, littérature et journalisme à travers le monde, Hartsock propose de distinguer ce qu'il appelle le journalisme littéraire $^{5}$, une forme qui serait spécifiquement américaine, et le reportage littéraire, propre à l'Europe - même si, dans son article, il ne discute que des exemples en langue allemande et russe. Selon Hartsock,

Literary reportage and literary journalism are much the same when they both emphasize narrative and descriptive modalities and eschew discursive polemic. But while literary journalism engages Bakhtin's inconclusive present, literary reportage historically has either done so or been co-opted by unambiguous ideology (again, what Bakhtin called « the distanced image of the absolute past» in which a response is prescribed instead of a phenomenal inconclusive present left open to interpretation). (Hartsock, 2011, p. 41)

Si l'on suit Hartsock, le journalisme littéraire américain relèverait donc toujours d'une modalité " narra-descriptive " et serait toujours ouvert à des interprétations multiples. Le reportage littéraire européen serait une forme plus élastique, pouvant correspondre au journalisme littéraire, mais pouvant aussi se présenter sous la forme d'un récit dépourvu d'ouverture interprétative, ou même opter pour une modalité plus discursive, avec une dimension polémique ou idéologique forte.

A priori, cette distinction peut étonner pour deux raisons : d'une part, il semble contre-intuitif que le reportage littéraire, qui

5 Correspondant à ce que l'on appelle ici journalisme narratif. 
n'est qu'un genre journalistique parmi d'autres, puisse prendre des formes plus diverses que l'ensemble du journalisme dit littéraire ; d'autre part, dans la tradition française en tout cas, le reportage est d'abord apparu comme l'un des nouveaux genres propres à une presse plus centrée sur les faits et est encore défini aujourd'hui comme un genre fondamentalement factuel. Si l'on trouve dans l'histoire du grand reportage en France de nombreux exemples de textes marqués idéologiquement, au point que l'on a pu parler de reportages « engagés » ou de reportages « de combat » (Martin, 2005), le fait même d'attacher un qualificatif au terme de reportage dans ces deux appellations semble indiquer que ce n'est pas là la nature fondamentale du genre.

Lorsque l'on s'intéresse aux deux noms souvent cités comme les grandes figures du grand reportage à la française, Joseph Kessel - surnommé 1'Empereur des grands reporters - et Albert Londres - le Prince des grands reporters -, le premier s'inscrit clairement dans la description et la narration factuelle, bien qu'en même temps éminemment subjective. Définissant le reportage comme « un roman d'aventures réel » (dans Boucharenc, 2004, p. 84), Kessel prend comme sujet de prédilection «l'homme, un type, un individu dans son micro-milieu, avec ses comportements, ses sentiments, ses ressorts intimes » (Martin, 2005, p. 251). Il écrit par exemple, dans « Les chasseurs d'esclaves », où il accompagne une caravane d'esclaves africains en route vers le Moyen-Orient :

Les yeux des esclaves étaient fixés sur nous. Je n'en ai jamais croisé qui fussent les truchements d'une pensée aussi rudimentaire. Seuls des yeux de ruminants doux, peureux et passifs, peuvent donner une idée de l'expression qu'avaient les grosses prunelles roulant à fleur du visage. Ces gens attendaient de nous tout le mal ou tout le bien, indifféremment. (Kessel, 2010, p. 27)

Si Kessel fait ressortir l'horreur de la situation des esclaves - « Ils avaient servi de réserve à bétail humain : les hommes pour le labeur, les femmes pour la jouissance des vainqueurs » (2010, p. 27) - et ne s'y montre clairement pas indifférent - « Je ne pus 
retenir un frisson » (2010, p. 32) -, il ne se lance jamais dans un débat sur la question de l'esclavage.

La figure d'Albert Londres est plus complexe. Il constitue, aujourd'hui encore, un symbole de l'indépendance journalistique - notamment pour la célèbre phrase citée dans le premier éditorial de XXI. Sa conception du journalisme est explicitée dans une autre de ses phrases restées célèbres : « Notre métier n'est pas de faire plaisir, non plus de faire du tort, il est de porter la plume dans la plaie » (dans Martin, 2005, p. 231).

À partir de 1923 et «Au bagne », Londres développe un reportage « à charge » : il dénonce à peu près tout ce qu'il a vu à Cayenne - les conditions de détention, le manque d'hygiène, les mauvais traitements, etc. Il devient « un journaliste "engagé", non pas comme le seront des intellectuels, mais comme quelqu'un qui supporte mal les injustices et les dévoile » (Eveno, 2011, p. 164). Il publie même, après le dernier épisode de son reportage, une lettre ouverte au ministre en charge des colonies réclamant qu'il prenne des mesures pour améliorer la vie des forçats. Cependant, le reportage en lui-même reste principalement narratif et descriptif. Le reporter y raconte son voyage, les endroits qu'il visite, les personnes qu'il rencontre et, s'inscrivant dans le paradigme de la « chose vue », ses réactions à tout cela. Quand, parfois, il bascule dans un registre plus éditorialisant, c'est toujours à partir de ce même paradigme : «A-t-on le droit, pour la même faute, de condamner un homme deux fois? Laissons la théorie. Regardons encore la réalité » (Londres, 1992, p. 92).

Londres apparaît finalement comme « un infatigable questionneur, toujours lucide face à ce que Paul Nizan appelait "les concepts dociles que rangent les caissiers soigneux de la pensée bourgeoise" "(Deluche, 2001, p. 46). Ses reportages sont même sujets à des interprétations conflictuelles. Joëlle Deluche, par exemple, soutient que le reporter «s'est battu pour l'idée d'humanité, afin d'inclure dans la grande famille commune tous les exclus » (2001, p. 46), tandis que Géraldine Muhlmann considère qu'il conserve « un curieux dégagement, un étonnement distant, un regard extérieur, faisant advenir l'étrangeté » (2004, p. 79) et maintient donc les exclus hors de la communauté que 
forment le journaliste et les lecteurs.

Par ailleurs, la posture de Londres et la production journalistique qui en découle ne semblent pas si différentes, dans leurs objectifs et leurs modalités, de celles de Nellie Bly, une des premières journalistes narratives américaines, lorsqu'elle décrit les dix jours qu'elle a passés dans un asile de fous - un sujet que Londres a d'ailleurs aussi exploré. Bly avait reçu pour mission : « to find out and describe its inside workings, which are always, so effectually hidden by white-capped nurses, as well as by bolts and bars, from the knowledge of the public » (Bly, 1887). Et elle note, en introduction à l'édition en livre de son reportage : « I have at least the satisfaction of knowing that the poor unfortunates will be the better cared for because of my work » (1887).

Tout au long de l'histoire du journalisme narratif américain, on peut repérer des textes qui comportent une certaine dimension polémique, une forme d'engagement ou une clôture interprétative plus marquée. Stephen Crane, qui décrit si bien les combats auxquels il assiste, note aussi, toujours à propos conflit grécoturc : " I hoped the Greeks on the plain would hurry and drive the Turks from their position » (1984, p. 937). James Agee explicite clairement le but politique de Let Us Now Praise Famous Men, considéré comme l'une des oeuvres les plus ouvertes de cette longue tradition : il espère que « the reader will be edified, and may feel kindly disposed toward any well-thought-out liberal efforts to rectify the unpleasant situation down South, and will somewhat better and more guiltily appreciate the new good meal he eats » (2001, p. 12).

Les écrits de Norman Mailer, tout en étant également ouverts sur le plan interprétatif, ne sont pas dépourvus d'une forme d'engagement polémique non plus, The Armies of the Night (2007) racontant par exemple sa participation aux manifestations contre le Pentagone en pleine guerre du Vietnam en 1968. Si, dans le Gonzo Journalism de Hunter Thompson, on ne sait souvent même pas discerner tout à fait ce qui relève des faits et de la fantaisie, on ne peut nier la critique sociale forte qui se dégage de son œuvre (notamment Hartsock, 2012). Plus récemment, dans Into the Wild (1997), Jon Krakauer juxtapose son expérience personnelle à 
celle d'un jeune américain mort loin de tout en Alaska pour mieux éclairer l'expérience de ce dernier.

Sans prétendre entrer ici dans le large débat sur les relations entre narration et rhétorique, il me semble nécessaire de se demander dans quelle mesure une dimension plus argumentative ou idéologique et une volonté de clôture interprétative ne seraient pas des tendances ou des tentations propres à la forme même du récit - et donc inhérentes au grand reportage français tout comme au journalisme narratif américain. Le contexte culturel et l'histoire du journalisme dans ce contexte particulier viendraient alors encourager ou, au contraire, freiner ces tentations - ce qui expliquerait pourquoi la dimension idéologique et la clôture interprétative seraient plus marquées dans certaines traditions journalistiques que dans d'autres. À nouveau, il s'agit là d'une hypothèse qu'il faudrait approfondir et qu'il pourrait être intéressant d'explorer dans d'autres sphères journalistiques.

\section{Conclusion}

Cet article, même s'il ne fait qu'esquisser quelques premiers ponts possibles entre le grand reportage à la française et le journalisme narratif américain ${ }^{6}$, éclaire à quel point, malgré l'originalité des mooks en tant que supports médiatiques matériels, ces revues s'inscrivent dans une longue histoire de résistance, au travers du récit, aux pratiques journalistiques dominantes résistance qui s'est développée aussi bien en France qu'aux États-Unis. En faisant apparaître plusieurs similitudes quant à la naissance, au développement et, dans une certaine mesure, à la nature même de ces deux traditions journalistiques, l'article met en évidence l'importance de développer une approche non seulement historique, mais aussi internationale, du recours au récit en journalisme.

Que les discours de revendication de cet héritage par les

6 La place de l'ironie, en son sens étroit comme au sens large de réflexivité et remise en question critique, dans ces deux traditions, ainsi que la question de leurs « ancêtres » tout au long du $19^{\mathrm{e}}$ siècle - deux autres points par rapport auxquels des liens sont également à faire - n'ont malheureusement pas pu être abordées ici, faute d'espace. 
mooks relèvent d'une véritable nostalgie pour un âge d'or perdu $\mathrm{du}$ journalisme, d'une tentative de légitimation au sein de la sphère journalistique par la référence au passé ou même d'un argument marketing dans un contexte où le « retour à » est à la mode, ils appellent une analyse plus approfondie des relations entre grand reportage français et journalisme narratif américain - et probablement d'autres traditions journalistiques narratives encore à travers le monde. Au-delà des discours des publications, il serait également intéressant, dans un deuxième temps, d'analyser la façon dont chaque mook se positionne concrètement, au travers des récits qu'il choisit de publier, par rapport à la dynamique entre cet héritage complexe et les bouleversements que connaît actuellement la sphère journalistique francophone.

\section{Références}

Agee, J., \& Evans, W. (2001). Let Us Now Praise Famous Men. Boston : Mariner Books.

Bak, J. S., \& Reynolds, B. (Éds.). (2011). Literary Journalism Across the Globe: Journalistic Traditions and Transnational Influences. Amherst : University of Massachusetts Press.

Bly, N. (1887). Ten Days in a Mad-House. Disponible à : http://digital.library.upenn. edu/women/bly/madhouse/madhouse.html

Boucharenc, M. (2004). L'écrivain-reporter au cœur des années trente. Villeneuve d'Ascq : Presses universitaires du Septentrion.

Boucharenc, M., \& Deluche, J. (Éds.). (2001). Littérature et reportage. Limoges : Presses universitaires de Limoges.

Boynton, R. (2005). The New New Journalism: Conversations with America's Best Nonfiction Writers on Their Craft. New York : Vintage.

Cahan, A. (1998). Can't get their minds ashore. In K. Kerrane \& B. Yagoda (Éds.), The Art of Fact: A Historical Anthology of Literary Journalism (pp. 76-79). New York : Touchstone.

Connery, T. (Éd.). (1992). A Sourcebook of American Literary Journalism: Representative Writers in an Emerging Genre. New York: Greenwood Press.

Crane, S. (1984). Crane at Velestino. In S. Crane, Prose and Poetry (pp. 934-938). New York : Library of America.

Delporte, C. (1995). Les journalistes dans l'entre-deux-guerres : Une identité en crise. Vingtième Siècle. Revue d'histoire, 47(1), 158-175. Disponible à : https://doi. org/10.3406/xxs.1995.3186

Deluche, J. (2001). Albert Londres, le premier des reporters heureux. In M. Boucharenc \& J. Deluche (Éds.), Littérature et reportage (pp. 31-46). Limoges : Presses universitaires de Limoges.

Durand, P. (2012). Le reportage. In D. Kalifa, P. Régnier, M.-E. Thérenty, \& A. Vaillant (Éds.), La civilisation du journal: Histoire culturelle et littéraire de la presse française au XIXe siècle (1800-1914) (pp. 1011-1024). Paris : Nouveau Monde. 
Eveno, P. (Éd.). (2011). Les grands articles qui ont fait l'histoire. Paris : Flammarion. Ferenczi, T. (1996). L'invention du journalisme en France. Paris : Payot.

Filteau, C. (2001). Gaston Leroux ou l'«obsession» de la démocratie. In M. Boucharenc \& J. Deluche, Littérature et reportage (pp. 301-319). Limoges : Presses universitaires de Limoges.

Hartsock, J. (2000). A History of American Literary Journalism The Emergence of a Modern Narrative Form. Amherst : University of Massachusetts Press.

Hartsock, J. (2011). Literary Reportage: The "Other" Literary Journalism. In J. S. Bak \& B. Reynolds (Éds.), Literary Journalism Across the Globe: Journalistic Traditions and Transnational Influences (pp. 23-46). Amherst : University of Massachusetts Press.

Hartsock, J. (Éd.). (2012). Fear and Loathing in Las Vegas + 40 [Special issue]. Literary Journalism Studies, 4(1).

Huret, J. (1901). Tout yeux, tout oreilles. Paris : Eugène Fasquelle.

Joignot, F. (2008). Jean-François Bizot: Portrait d'un homme de presse en artiste. Médiamorphoses, (HS 4), 113-117.

Kalifa, D., Régnier, P., Thérenty, M.-E., \& Vaillant, A. (2012). La civilisation du journal : Histoire culturelle et littéraire de la presse française au XIXe siècle (18001914). Paris : Nouveau Monde.

Keeble, R., \& Tulloch, J. (Éds.). (2012). Global Literary Journalism: Exploring the Journalistic Imagination. New York : Peter Lang.

Keeble, R., \& Tulloch, J. (Éds.). (2014). Global Literary Journalism: Exploring the Journalistic Imagination (Volume 2). New York : Peter Lang.

Kervran, P., \& Kien, A. (2010). Les années Actuel: Contestations rigolardes et aventures modernes. Marseille : Le Mot et le Reste.

Kessel, J. (2010). Les jours de l'aventure. Paris : Tallandier.

Krakauer, J. (1997). Into the Wild. New York : Anchor.

Lits, M. (2008). Du récit au récit médiatique. Bruxelles : De Boeck.

Londres, A. (1992). CEuvres complètes. Paris : Arléa.

Mailer, N. (2007). The Armies of the Night: History as a Novel, the Novel as History. New York : Plume.

Martin, M. (2005). Les grands reporters : Les débuts du journalisme moderne. Paris : Louis Audibert.

Muhlmann, G. (2004). Une histoire politique du journalisme : XIX $X^{e}-X X^{e}$ siècle. Paris : Presses universitaires de France.

Pauly, J. (2014). The New Journalism and the struggle for interpretation. Journalism, 15(5), 589-604.

Roggenkamp, K. (2005). Narrating the News: New Journalism and Literary Genre in Late Nineteenth-century American Newspapers and Fiction. Kent : Kent State University Press.

Ruellan, D. (2007). Le journalisme ou le professionnalisme du flou. Grenoble : Presses universitaires de Grenoble.

Schudson, M. (1978). Discovering the News: A Social History of American Newspapers. New York : Basic Books.

Sims, N. (2007). True Stories: A Century of Literary Journalism. Evanston : Northwestern University Press.

Sims, N. (Éd.). (2008). Literary journalism in the twentieth century. Evanston : Northwestern University Press. 
Thérenty, M.-E. (2007). La littérature au quotidien : Poétiques journalistiques au XIXe siècle. Paris : Seuil.

Thérenty, M.-E., \& Vaillant, A. (2004). Presse et plumes: journalisme et littérature au $X I X^{e}$ siècle. Paris : Nouveau monde.

Vanoost, M. (2013). Journalisme narratif : proposition de définition, entre narratologie et éthique. Les Cahiers Du Journalisme, (25), 140-161.

Wolfe, T. (Éd.). (1975). The New Journalism. New York : Harper \& Row.

\section{(9) $\Theta \Theta$}

Publié sous la licence Creative Commons

«Attribution - Pas d'Utilisation Commerciale - Pas de Modification 4.0 International» (CC BY-NC-ND) 